\title{
Amplitude analysis of the anomalous decay $\eta^{\prime} \rightarrow \pi^{+} \pi^{-} \gamma$
}

\author{
Ling-Yun Dai, ${ }^{1,2, *}$ Xian-Wei Kang, ${ }^{3,4, \dagger}$ Ulf-G. Meißner, ${ }^{5,2, \star}$ Xin-Ying Song, ${ }^{6,7,8}$ and De-Liang Yao, ${ }^{8, \|}$ \\ ${ }^{1}$ School of Physics and Electronics, Hunan University, Changsha 410082, China \\ ${ }^{2}$ Institute for Advanced Simulation, Institut für Kernphysik and Jülich Center for Hadron Physics, \\ Forschungszentrum Jülich, D-52425 Jülich, Germany \\ ${ }^{3}$ College of Nuclear Science and Technology, Beijing Normal University, Beijing 100875, China \\ ${ }^{4}$ Institute of Physics, Academia Sinica, Taipei 115, Taiwan \\ ${ }^{5}$ Helmholtz Institut für Strahlen- und Kernphysik and Bethe Center for Theoretical Physics, \\ Universität Bonn, D-53115 Bonn, Germany \\ ${ }^{6}$ Institute für Kernphysik, Forschungszentrum Jülich, D-52425 Jülich, Germany \\ ${ }^{7}$ Institute of High Energy Physics, Beijing 100049, China \\ ${ }^{8}$ Instituto de Física Corpuscular (centro mixto CSIC-UV), Institutos de Investigación de Paterna, \\ Apartado 22085, 46071 Valencia, Spain
}

(Received 11 December 2017; published 13 February 2018)

\begin{abstract}
In this paper we perform an amplitude analysis of $\eta^{\prime} \rightarrow \pi^{+} \pi^{-} \gamma$ and confront it with the latest BESIII data. Based on the final-state interaction theorem, we represent the amplitude in terms of an Omnés function multiplied by a form factor that corresponds to the contributions from left-hand cuts and right-hand cuts in the inelastic channels. We also take into account the isospin violation effect induced by $\rho-\omega$ mixing. Our results show that the anomaly contribution is mandatory in order to explain the data. Its contribution to the decay width of $\Gamma\left(\eta^{\prime} \rightarrow \pi \pi \gamma\right)$ is larger than that induced by isospin violation. Finally we extract the pole positions of the $\rho$ and $\omega$ as well as their corresponding residues.
\end{abstract}

DOI: $10.1103 /$ PhysRevD.97.036012

\section{INTRODUCTION}

There has long been an interest in the study of anomalous decays, which are driven by the chiral anomaly of QCD. ${ }^{1}$ The $\eta^{\prime} / \eta \rightarrow \pi^{+} \pi^{-} \gamma$ decays are typical processes for exploring the box anomaly and investigating the $\rho-\omega$ mixing mechanism. They are useful to extract the pion vector form factor [2-5] and the form factors of $\eta^{\prime} / \eta \rightarrow \gamma \gamma^{*}$ transitions [3-5], helping us to further test, e.g., the Pascalutsa-Vanderhaeghen lightby-light sum rule [6-8]. The so-obtained knowledge of these form factors is also crucial in the determination of the hadronic contribution to the anomalous magnetic moment of the muon [9-11], as witnessed by the preparation for the planned experiments at Fermilab [12] and J-PARC [13]. Furthermore, the two processes are helpful for decoding the information on the resonances (intermediate states) such as $\rho$

\footnotetext{
1.dai@fz-juelich.de

${ }^{\dagger}$ kangxianwei1@gmail.com

${ }^{*}$ meissner@hiskp.uni-bonn.de

$\S$ x.song@fz-juelich.de

deliang.yao@ific.uv.es

${ }^{\mathrm{I}}$ For an introduction on anomalies, see e.g. [1].
}

Published by the American Physical Society under the terms of the Creative Commons Attribution 4.0 International license. Further distribution of this work must maintain attribution to the author(s) and the published article's title, journal citation, and DOI. Funded by SCOAP ${ }^{3}$. and $\omega$. For instance, the branching ratio of $\omega \rightarrow \pi \pi$ has been extracted in Ref. [5].

Searching for the box anomaly in the $\eta^{\prime} / \eta \rightarrow \pi^{+} \pi^{-} \gamma$ decays is also an interesting topic on the experimental side. For a $\eta$ decay, WASA-at-COSY [14] and KLOE [15] have determined the relevant parameters using the approach, proposed in Ref. [2], based on chiral perturbation theory $(\chi \mathrm{PT})$ and dispersion theory. For the corresponding $\eta^{\prime}$ decay, JADE [16], CELLO [17], PLUTO [18], TASSO [19], TPC [20], and ARGUS [21] all observed a peak shift of about $+20 \mathrm{MeV} / c^{2}$ in the dipion mass spectrum, with respect to the expected position of the $\rho^{0}$. This certainly indicates that only a contribution from the $\rho$ is not sufficient. This issue is also discussed in Ref. [22] for $B_{\ell 4}$ decay. In the analysis of Ref. [23], it is shown that the contribution from the box anomaly could be essential in $\eta^{\prime} \rightarrow \pi^{+} \pi^{-} \gamma$. Later, the significance of the box anomaly was found to be $4 \sigma$ by the Crystal Barrel (CB) Collaboration [24] with 7400 events, while the L3 Collaboration [25] claimed that the $\rho$ contribution is sufficient to describe the data with less data $(2123 \pm 53$ events). Nonetheless, the CB data is not precise enough to disentangle the effect of the $\rho-\omega$ interference from others in the line shape, as shown, e.g., by Ref. [26]. Recently, the BESIII Collaboration [27,28] explored the process $\eta^{\prime} \rightarrow$ $\pi^{+} \pi^{-} \gamma$ with very high statistics (of about $9.7 \times 10^{5}$ events), and the $\rho-\omega$ interference is seen for the first time in this 
decay. Therefore, it is timely to make a refined amplitude analysis of the anomalous decay $\eta^{\prime} \rightarrow \pi^{+} \pi^{-} \gamma$.

In the $\eta^{\prime} \rightarrow \pi^{+} \pi^{-} \gamma$ decay, the contribution of the anomaly is significant and hence can not be simply determined by a tree-level amplitude from the WessZumino-Witten (WZW) [29,30] term. In Ref. [31], this anomalous decay is studied using $\chi \mathrm{PT}$ in combination with a nonperturbative method based on coupled channels. Here, by properly taking into account the effects of the final-state interaction (FSI) and $\rho-\omega$ mixing, we aim at obtaining precise information about the anomaly. On the one hand, since the effect of three-body rescattering between the pions and the photon is negligible due to the tiny electromagnetic interaction, a purely strong $\pi \pi$ FSI should be sufficient. The nonperturbative $\pi \pi$ FSI is implemented in a model-independent way, where the contribution corresponding to the unitary cut is represented by an Omnès function. On the other hand, our treatment of $\rho-\omega$ mixing is beyond the simplified version employed in Ref. [5]. In our case, the isospin-violating $\rho-\omega$ interference is constructed by invoking the resonance chiral theory $(\mathrm{R} \chi \mathrm{T})$ [32-38] (for earlier attempts using $\chi \mathrm{PT}$ with explicit vector mesons, see e.g., Refs. $[39,40])$. The approach we use here is a combination of the dispersion theory and chiral effective field theory. Similar prescriptions have been widely applied to, e.g., $\gamma \gamma \rightarrow \pi \pi$ [41,42], $\eta \rightarrow 3 \pi$ [43], $\phi / \omega \rightarrow \pi \gamma^{*}$ [44], $D_{\ell 3}$ [45] and $B_{\ell 4}$ [22]. With our method, it is clearly shown that the anomaly is mandatory in order to describe the experimental data. Besides, it is possible and also interesting to see how much the leading-order (LO) result in the $1 / N_{C}$ expansion of $\mathrm{R} \chi \mathrm{T}$ is modified by the $\pi \pi$ rescattering. For a general discussion of the role of vector mesons in anomalous processes, see e.g. [46,47].

This paper is organized as follows. In Sec. II we discuss the formalism for the $\eta^{\prime} \rightarrow \pi \pi \gamma$ decay amplitude. The inclusion of the FSI effect is discussed in Sec. II A, while the isospin-violating form factors are calculated within $\mathrm{R} \chi \mathrm{T}$ in Sec. II B. Section II C provides the final form of the decay amplitude. Section III contains our numerical results. We fit to the experimental data and pin down all the relevant unknown parameters in Sec. III A. In Sec. III B, we extract the poles of the $\rho$ and $\omega$ resonances as well as their couplings to the $\eta^{\prime} \gamma$ states, which are then used to calculate the decay widths. We also discuss the impact of the isospinviolating effect on the $P$-wave phase of $\pi^{+} \pi^{-}$scattering. Finally, we summarize and make conclusions in Sec. IV. The explicit expressions of the isospin-violating form factors in $\mathrm{R} \chi \mathrm{T}$ are relegated to the Appendix.

\section{AMPLITUDE FORMALISM}

\section{A. Final state interaction}

Bose and charge conjugation symmetry guarantee that $\eta^{\prime} \rightarrow \pi^{0} \pi^{0} \gamma$ is forbidden, and hence we only need to consider the mode $\eta^{\prime} \rightarrow \pi^{+} \pi^{-} \gamma$. As will be explained below, its full amplitude is composed of partial waves with an isospin $I=1$ and an odd angular momentum, i.e. $P, F, \ldots$ waves. The isospin violation part induced mainly by the $\rho-\omega$ mixing is of an isospin $I=0$, which will be discussed in the next section. The Lorentz-invariant decay amplitude for $\eta^{\prime}(q) \rightarrow$ $\pi^{+}\left(p_{+}\right) \pi^{-}\left(p_{-}\right) \gamma(k)$ can be written as

$$
\mathcal{M}_{\lambda}=e \epsilon^{\mu \nu \alpha \beta} \epsilon_{\mu}(k, \lambda) q_{\nu} p_{\alpha}^{+} p_{\beta}^{-} \mathcal{F}_{\lambda}(s, \cos \theta),
$$

where $\epsilon_{\mu}(k, \lambda)$ is the polarization of the outgoing photon with an helicity $\lambda$. The variables of the form factor $\mathcal{F}_{\lambda}$ are chosen to be $s \equiv(q-k)^{2}$ and $\cos \theta$. Here, $\theta$ is the scattering angle in the $\pi \pi$ center of mass frame. The partial-wave decomposition of $M_{\lambda}$ reads

$$
\mathcal{M}_{\lambda}=16 \pi \sqrt{N_{\pi \pi}} \sum_{J} M_{J \lambda}(s) d_{\lambda 0}^{J}(\theta)(2 J+1),
$$

where the normalization factor should be set to $N_{\pi \pi}=2$, in accordance with the Bose statistics of identical particles. The isospin decomposition is given by $M_{J \lambda}(s)=$ $-M_{J \lambda}^{1}(s) / \sqrt{2}$, so that

$$
M_{J \lambda}^{1}(s)=-\frac{e\left(M_{\eta^{\prime}}^{2}-s\right) \sqrt{s-4 M_{\pi}^{2}} F_{J \lambda}^{1}(s)}{128 \sqrt{2} \pi},
$$

with

$$
F_{J \lambda}^{1}(s)=\int_{-1}^{1} d \cos \theta \mathcal{F}_{\lambda}(s, \cos \theta) \sin \theta d_{\lambda 0}^{J}(\theta) .
$$

From the parity conservation one has $F_{J \lambda}^{1}(s)=$ $(-)^{J} F_{J(-\lambda)}^{1}(s)$. Here we only keep the lowest $P$-wave and ignore $F$ - and higher partial waves since their contributions are relatively small. Following these constraints we have only one independent partial wave, $M_{1+}^{1}(s)$ or $M_{1-}^{1}(s)$. Comparing Eqs. (1)-(4) one soon finds $\mathcal{F}_{+}(s, \cos \theta)=$ $-3 /(2 \sqrt{2}) F_{1+}^{1}(s)$. Notice that higher order corrections of QED are negligible compared to the hadronic FSI; thus we do not take them into account. Finally we construct our amplitude based on Watson's FSI theorem,

$$
F_{1+}^{1}(s)=P(s) \Omega_{1}^{1}(s),
$$

with $\Omega_{1}^{1}(s)$ the so-called Omnès function and $P(s)$ a polynomial. We will discuss $P(s)$ in Sec. II C. The Omnès function satisfies the following dispersion relation:

$$
\Omega_{1}^{1}(s)=\exp \left(\frac{s}{\pi} \int_{4 M_{\pi}^{2}}^{\infty} d s^{\prime} \frac{\varphi_{1}^{1}\left(s^{\prime}\right)}{s^{\prime}\left(s^{\prime}-s\right)}\right) .
$$

The function $\varphi_{1}^{1}(\mathrm{~s})$ denotes the phase of a $P$-wave elastic $\pi \pi \rightarrow \pi \pi$ amplitude, which was given in a previous amplitude analysis of $\pi \pi$ scattering $[48,49]$. In Fig. 1, we show the phase and modulus of the Omnès function. Up to the $\bar{K} K$ threshold, it has the same phase as that of the decay amplitude $F_{1+}^{1}(s)$.

\section{B. Formalism of $\mathbf{R} \chi \mathbf{T}$}

In this section, we will calculate the chiral anomaly contribution as well as the isospin-violating amplitude for $\eta^{\prime} \rightarrow \pi^{+} \pi^{-} \gamma$ at the LO in the large $N_{C}$ expansion in $\mathrm{R} \chi \mathrm{T}$, 

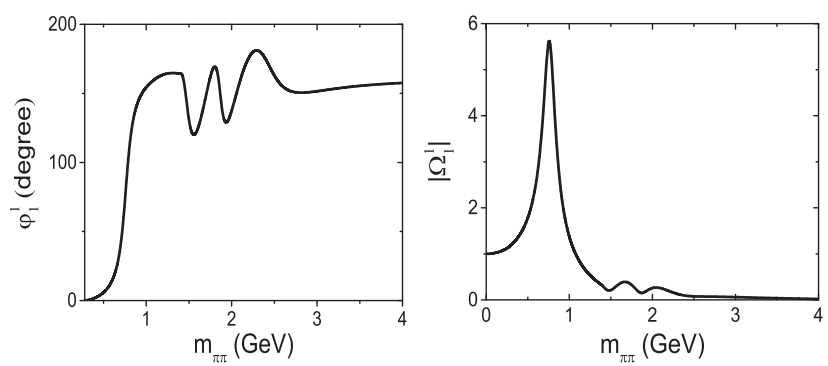

FIG. 1. Left: Phase of the $\pi \pi$ scattering amplitude in a $P$-wave with isospin $I=1$. Right: a modulus of the Omnès function.

with $N_{C}$ the number of colors. The $\rho-\omega$ mixing is taken into account following Ref. [38]. The relevant chiral Lagrangian can be written as [34],

$$
\mathcal{L}_{R \chi T}=\mathcal{L}_{\text {kin }}^{\mathrm{v}}+\mathcal{L}_{\text {int }}^{\mathrm{v}}+\mathcal{L}_{(4)}^{\mathrm{GB}} .
$$

The part $\mathcal{L}_{(4)}^{\mathrm{GB}}$ containing the LO operators of the chiral anomaly is $[29,30]$

$$
\mathcal{L}_{(4)}^{\mathrm{GB}}=i \frac{N_{C} \sqrt{2}}{12 \pi^{2} F^{3}} \varepsilon_{\mu \nu \rho \sigma}\left\langle\partial^{\mu} \Phi \partial^{\nu} \Phi \partial^{\rho} \Phi v^{\sigma}\right\rangle+\cdots,
$$

where $F \approx 92.2 \mathrm{MeV}$ is the pion decay constant in the chiral limit and $\Phi$ is a nonet matrix collecting the pseudoscalar Goldstone bosons,

$$
\Phi=\left(\begin{array}{ccc}
\frac{\pi^{0}}{\sqrt{2}}+\frac{\eta_{8}}{\sqrt{6}} & \pi^{+} & K^{+} \\
\pi^{-} & -\frac{\pi^{0}}{\sqrt{2}}+\frac{\eta_{8}}{\sqrt{6}} & K^{0} \\
K^{-} & \bar{K}^{0} & -\frac{2 \eta_{8}}{\sqrt{6}}
\end{array}\right)+\frac{\mathbb{1}}{\sqrt{3}} \eta_{0} .
$$

The mixing of the $\eta_{8}$ and $\eta_{0}$ with an angle $\theta_{P}$ yields the physical $\eta$ and $\eta^{\prime}$ states. $^{2}$

$$
\left(\begin{array}{l}
\eta_{8} \\
\eta_{0}
\end{array}\right)=\left(\begin{array}{cc}
\cos \theta_{P} & \sin \theta_{P} \\
-\sin \theta_{P} & \cos \theta_{P}
\end{array}\right)\left(\begin{array}{c}
\eta \\
\eta^{\prime}
\end{array}\right) .
$$

The kinematic part of the vector resonances reads

$$
\mathcal{L}_{\text {kin }}^{\mathrm{v}}=-\frac{1}{2}\left\langle\nabla^{\lambda} V_{\lambda \mu} \nabla_{\nu} V^{\nu \mu}\right\rangle+\frac{1}{4} M_{V}^{2}\left\langle V_{\mu \nu} V^{\mu \nu}\right\rangle,
$$

where $M_{V}$ is the mass of the vector resonances in the chiral limit. The matrix field $V^{\mu \nu}$, in antisymmetric tensor representation, incorporates the low-lying vector resonances in a nonet form

$$
V^{\mu \nu}=\sum_{i=1}^{8} \frac{\lambda_{i}}{\sqrt{2}} V_{i}^{\mu \nu}+\frac{\mathbb{1}}{\sqrt{3}} V_{0}^{\mu \nu},
$$

\footnotetext{
${ }^{2} \mathrm{We}$ notice that more complicated schemes for $\eta-\eta^{\prime}$ mixing involving two mixing angles have been studied in e.g., Refs. [50-54], which is commonly used to describe the twophoton decays well $[50,51,54]$.
}

with $\lambda_{i}(i=1, \ldots, 8)$ and $\mathbb{1}$ the standard Gell-Mann matrices and the $3 \times 3$ unit matrix, respectively. The covariant derivative acting on the vector fields is defined by [32]

$$
\begin{aligned}
\nabla_{\alpha} V^{\mu \nu} & =\partial_{\alpha} V^{\mu \nu}+\left[\Gamma_{\alpha}, V^{\mu \nu}\right], \\
\Gamma_{\mu} & =\frac{1}{2}\left(u^{\dagger}\left(\partial_{\mu}-r_{\mu}\right) u+u\left(\partial_{\mu}-l_{\mu}\right) u^{\dagger}\right), \\
l_{\mu} & =v_{\mu}-a_{\mu}, \\
r_{\mu} & =v_{\mu}+a_{\mu},
\end{aligned}
$$

where $v_{\mu}$ and $a_{\mu}$ denote external vector and axial-vector fields, respectively. It is worth noting that the photon field $A_{\mu}$ can be introduced by setting $r_{\mu}=l_{\mu}=e Q A_{\mu}$ with $Q=$ $\operatorname{diag}\{2 / 3,-1 / 3,-1 / 3\}$. Furthermore, $u=\exp \{i \Phi /(\sqrt{2} F)\}$.

The interaction between the vector resonances and the Goldstone bosons is described by

$$
\mathcal{L}_{\mathrm{int}}^{\mathrm{v}}=\mathcal{L}_{(2)}^{\mathrm{v}}+\mathcal{L}_{(4)}^{\mathrm{v}}+\mathcal{L}_{(2)}^{\mathrm{vV}} .
$$

Here, the subscripts denote the chiral orders, the corresponding superscripts imply the numbers of vector resonance. Specifically, the first term reads [32]

$$
\mathcal{L}_{(2)}^{\mathrm{v}}=\frac{F_{V}}{2 \sqrt{2}}\left\langle V_{\mu \nu} f_{+}^{\mu \nu}\right\rangle+i \frac{G_{V}}{\sqrt{2}}\left\langle V_{\mu \nu} u^{\mu} u^{\nu}\right\rangle
$$

Here, $F_{V}$ and $G_{V}$ are unknown coupling constants, that can be fixed from certain resonance decays or from shortdistance QCD constraints, see in the Appendix. The chiral building blocks, $f_{+}^{\mu \nu}$ and $u_{\mu}$ are given explicitly in Ref. [32]. The last two terms in Eq (14) are of an odd-intrinsic parity. The pieces relevant to our calculation can be expressed as $[34,37]$

$$
\begin{aligned}
\mathcal{L}_{(4)}^{\mathrm{V}} & =\sum_{i=1}^{7} \frac{c_{i}}{M_{V}} \mathcal{O}_{\mathrm{VJP}}^{i}+\sum_{j=1}^{5} \frac{g_{j}}{M_{V}} \mathcal{O}_{\mathrm{VPPP}}^{j}, \\
\mathcal{L}_{(2)}^{\mathrm{VV}} & =\sum_{k=1}^{4} d_{k} \mathcal{O}_{\mathrm{VVP}}^{k} .
\end{aligned}
$$

These couplings are defined to be dimensionless. For the explicit expressions of the odd-intrinsic chiral operators we refer the readers to Refs. [34,37]. The values of the parameters, $c_{i}, g_{j}$ and $d_{k}$, are taken from Ref. [38] and are also given in our Appendix.

The fields used in the above-mentioned chiral effective Lagrangians are convenient for analyzing transformation properties under the chiral group. Nonetheless, not all of them directly correspond to physical states. In practice, the physical $\omega(782)$ and $\phi(1020)$ states are related to the octet and singlet components by a mixing angle $\theta_{V}$ through 

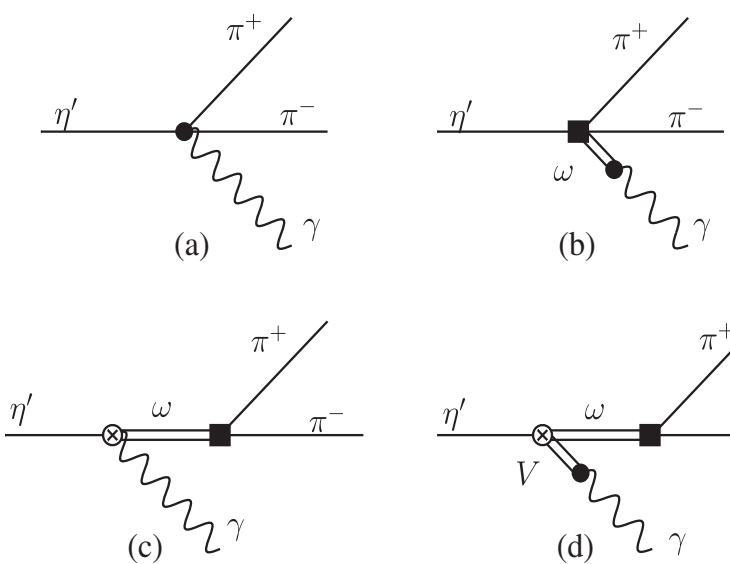

(c) $2 \gamma$

(d) $2 \gamma$

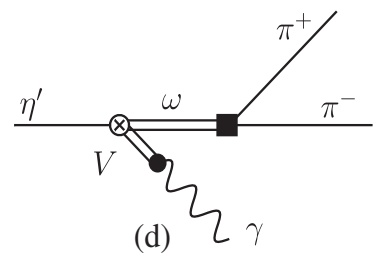

FIG. 2. The Feynmann diagrams of the Wess-Zumino-Witten (WZW) term (a), and the isospin violation amplitudes (b)-(d). The symbol "V" represents the vector resonances $\rho, \omega$, and $\phi$. The oddintrinsic parity and isospin-violating vertices are denoted by the circled crosses and black squares, respectively. Note that in (b) the black square also represents an odd-intrinsic parity vertex.

$$
\left(\begin{array}{c}
V^{8} \\
V^{0}
\end{array}\right)=\left(\begin{array}{cc}
\cos \theta_{V} & \sin \theta_{V} \\
-\sin \theta_{V} & \cos \theta_{V}
\end{array}\right)\left(\begin{array}{c}
\phi \\
\omega
\end{array}\right)
$$

In the same manner, the $\rho-\omega$ mixing due to isospin symmetry violation can be parametrized as

$$
\left(\begin{array}{c}
\bar{\rho}^{0} \\
\bar{\omega}
\end{array}\right)=\left(\begin{array}{cc}
\cos \delta & \sin \delta \\
-\sin \delta & \cos \delta
\end{array}\right)\left(\begin{array}{c}
\rho^{0} \\
\omega
\end{array}\right)
$$

with $\delta$ the mixing angle. The values of these mixing angles we used are listed in the Appendix ${ }^{3}$;.

Eventually, we are in the position to calculate the amplitudes for $\eta^{\prime} \rightarrow \pi^{+} \pi^{-} \gamma$ including explicitly the WZW term as well as the isospin violation. Throughout, we assume that the $\rho$ - $\omega$ mixing is the dominant isospin breaking effect. The relevant Feynman diagrams are displayed in Fig. 2, and the resulting amplitudes are given in the Appendix.

\section{Isospin-violating form factor}

To include the dominant isospin-violating effect, we utilize the following form for the polynomial in Eq. (5):

$$
P(s)=\alpha_{0}+\alpha_{1}\left(s-4 M_{\pi}^{2}\right)+P^{i \cdot v \cdot}(s) .
$$

The contributions from left-hand cuts (1.h.c) and inelastic right-hand cuts (r.h.c) are ascribed to the polynomial in front, and $P^{i . v .}(s)$ stands for the contribution from isospin violation. Following the experimental paper [27], we set

\footnotetext{
${ }^{3}$ We notice that a more complicated scheme for the $\rho-\omega$ mixing with two parameters has been studied in e.g., Refs. [55].
}

$$
\begin{aligned}
& P^{i . v \cdot}(s)=\beta_{0} F_{\text {tree }}^{i \cdot v \cdot}(s), \\
& F_{1+}^{i \cdot v \cdot}(s)=P^{i \cdot v \cdot}(s) \Omega_{1}^{1}(s),
\end{aligned}
$$

with $F_{\text {tree }}^{i . v}(s)$ the isospin-violating form factor given in Eq. (A3). The phase of $\pi \pi$ rescattering is included by the Omnès function, and the 1.h.c and inelastic r.h.c contributions of the isospin violation are absorbed in the parameter $\beta_{0}$. In principle there should be more terms rather than a single $\beta_{0}$, but in practice we find that one parameter is good enough to describe the data well.

We notice that $F_{\text {tree }}^{i, v}(s)$ has a sizeable imaginary part around $\sqrt{s}=M_{\omega}$. It modifies the $\pi^{+} \pi^{-}$phase in the vicinity of $\sqrt{s}=M_{\omega}$. Nevertheless, this is helpful for us to obtain $\rho-\omega$ mixing exactly in the $\pi^{+} \pi^{-}$FSI. Previous dispersive analyses [56,57] and also experiments [58-60] take only the contribution of $\rho$ in their partial wave analysis; thus the information of the isospin-violating part, i.e. the contribution from the $\omega$, is lacking in this region. This will be discussed with more details in the next section.

For the isospin-violating form factor $F_{\text {tree }}^{i . v}(s)$, we only need to calculate the diagrams, (b), (c) and (d), in Fig. 2. The results are given in the Appendix. Finally, the total amplitude is given by

$F_{1+}^{1}(s)=\left[\alpha_{0}+\alpha_{1}\left(s-4 M_{\pi}^{2}\right)+\beta_{0} F_{\text {tree }}^{i . v .}(s)\right] \Omega_{1}^{1}(s)$.

Note that $F_{1+}^{1}(s)$ contains an isospin-violating part $(I=0)$, though its superscript is labeled by "1", corresponding to $I=1$. With this amplitude one can get the formula for the dipion mass spectrum,

$$
\frac{d \Gamma}{d \sqrt{s}}=\frac{3 \alpha s^{3 / 2} \rho(s)^{3}\left(M_{\eta^{\prime}}^{2}-s\right)^{3}\left|F_{1+}^{1}(s)\right|^{2}}{2048 \pi^{2} M_{\eta^{\prime}}^{3}},
$$

with $\rho(s)=\sqrt{1-4 M_{\pi}^{2} / s}$. When fitting to the invariant mass spectrum of BESIII [27], we need to multiply it by a normalization factor $N$. In Ref. [3], the anomaly is obtained at the point where $s=t=u=0$, in the chiral limit. Similarly, we define the anomaly as $-e \mathcal{F}_{+}(0, \cos \theta)$. Notice that here $\theta$ is a dummy variable in $\mathcal{F}_{+}(0, \cos \theta)$. We have

$$
A=\frac{3 e}{2 \sqrt{2}}\left(\alpha_{0}-4 M_{\pi}^{2} \alpha_{1}+\beta_{0} F_{\text {tree }}^{i . v .}(0)\right),
$$

where $F_{\text {tree }}^{i . v}(0)=0.155 \mathrm{GeV}^{-3}$, see in the Appendix.

\section{NUMERICAL RESULTS}

\section{A. Fit to experimental data}

In this section, we fit to the invariant dipion mass spectrum by BESIII [27]. The decay width of $\eta^{\prime} \rightarrow$ $\pi^{+} \pi^{-} \gamma$ provided by the Particle Data Group (PDG) [61] is implemented in our fit to constrain the unknown normalization factor $N$. The following fits are performed: 
TABLE I. Results for the different fits explained in the text. The $\chi_{\text {average }}^{2}$ is the total $\chi^{2}$ of the invariant mass spectrum [27] divided by the number of data points. The PDG [61] value of $\Gamma\left(\eta^{\prime} \rightarrow\right.$ $\left.\gamma \pi^{+} \pi^{-}\right)$is $57.3 \pm 1.0 \mathrm{keV}$. The uncertainty is given from the fit.

\begin{tabular}{lccc}
\hline \hline & Fit 1 & Fit 2 & Fit 3 \\
\hline$\alpha_{0}\left(\mathrm{GeV}^{-3}\right)$ & $17.91 \pm 0.23$ & 14.37 & $18.41 \pm 0.19$ \\
$\alpha_{1}\left(\mathrm{GeV}^{-5}\right)$ & $11.78 \pm 0.18$ & $10.29 \pm 0.12$ & $12.37 \pm 0.17$ \\
$\beta_{0}$ & $\cdots$ & $0.132 \pm 0.002$ & $0.150 \pm 0.002$ \\
$\mathrm{~N}\left(\times 10^{8}\right)$ & $0.852 \pm 0.021$ & $1.25 \pm 0.07$ & $0.788 \pm 0.016$ \\
$\chi_{\text {average }}^{2}$ & 12.3 & 2.66 & 1.74 \\
$\Gamma_{\eta^{\prime} \rightarrow \gamma \pi^{+} \pi^{-}}(\mathrm{keV})$ & $57.3 \pm 8.6$ & $38.9 \pm 8.2$ & $62.0 \pm 5.9$ \\
anomaly $\left(\mathrm{GeV}^{-3}\right)$ & $5.46 \pm 0.42$ & $4.36 \pm 0.50$ & $5.61 \pm 0.29$ \\
\hline \hline
\end{tabular}

(1) Fit 1: We ignore the isospin violation $\left(\beta_{0}=0\right)$. The best-fit parameters are collected in the second column of Table I.

(2) Fit 2: We include the contribution of the isospin violation, and $\alpha_{0}$ is fixed by Eq. (24). The results are shown in the third column in Table I.

(3) Fit 3: As in fit 2 we include the isospin violation, but set $\alpha_{0}$ to be a free parameter. The results are shown in the fourth column in Table I.

In fact, a chiral matching can be imposed to fix $\alpha_{0}$ [62]. At low energies, our amplitude is required to coincide with the one calculated from the LO WZW term $[29,30]$. The corresponding Feynman diagram is shown in Fig. 2(a), and its form factor is given in Eq. (A2). We choose $s=4 M_{\pi}^{2}$ as the matching point to avoid any complication caused by the $\alpha_{1}$ term in Eq. (19). After the chiral matching described above, one obtains

$$
\alpha_{0}=\frac{\sqrt{2} N_{C}}{18 \sqrt{3} \pi^{2} F^{3} \Omega_{1}^{1}\left(4 M_{\pi}^{2}\right)}\left(\sin \theta_{P}+\sqrt{2} \cos \theta_{P}\right) .
$$

The value of $\alpha_{0}$ is fixed to be $14.37 \mathrm{GeV}^{-3}$ provided $\theta_{P}=$ $-21.37^{\circ}$. Note that $\Omega_{1}^{1}\left(4 M_{\pi}^{2}\right)=1.159$ is a real number. This corresponds to fit 2 . In contrast, if we use the double-anglesmixing scheme (DAMS), we obtain $\alpha_{0}=15.17$, where the angles and decay constants are taken from "NNLO fit-A" of Ref. [53]. In this case $\alpha_{0}$ is fairly increased and still faraway from that of fit 3 . In the absence of the high statics data of $\eta \rightarrow \pi^{+} \pi^{-} \gamma$, one can not reach a definite conclusion on how DAMS will improve the calculation.

Actually, one may treat $\theta_{P}$ as a free parameter, while $\alpha_{0}$ is always fixed by using Eq. (24) during the fit procedure. A good fit can be obtained with $\theta_{P} \approx-10.9 \pm 0.5^{\circ}$, which is compatible with the determinations in Refs. [3,63-65]. However, the resulting decay widths involving $\eta^{\prime}, \Gamma_{\eta^{\prime} \rightarrow \rho \gamma}$, $\Gamma_{\eta^{\prime} \rightarrow \omega \gamma}$ and $\Gamma_{\phi \rightarrow \eta^{\prime} \gamma}$, are now deviated about 30\%-60\% from the previous determinations in Ref. [38]. Thus, we fix $\theta_{P}$ at $-21.37^{\circ}$ [38] and set $\alpha_{0}$ free. This is fit 3. Note that here Eq. (24) is not implemented as a constraint. In fit 3 the BESIII data can be well described and, furthermore, the previous results in Ref. [38] for the above-mentioned decay widths are untouched. For comparison, the invariant mass spectrum, based on the fitted values of the parameters from fit 1, fit 2 and fit 3, are shown simultaneously in Fig. 3. Note that the Crystal Ball data points are superimposed on the plot.

For fit 1, the invariant mass spectrum is represented by the blue dotted line in Fig. 3. Obviously, there is no $\rho-\omega$ mixing structure appearing in the energy region $\sqrt{s} \in[0.76,0.8] \mathrm{GeV}$. This is not surprising as we do not take the isospin violation into account. The resulting decay width of $\eta^{\prime} \rightarrow \pi^{+} \pi^{-} \gamma$ is $57.3 \pm 8.6 \mathrm{keV}$ is absolutely in agreement with the value given by PDG. For fit 2, the fit

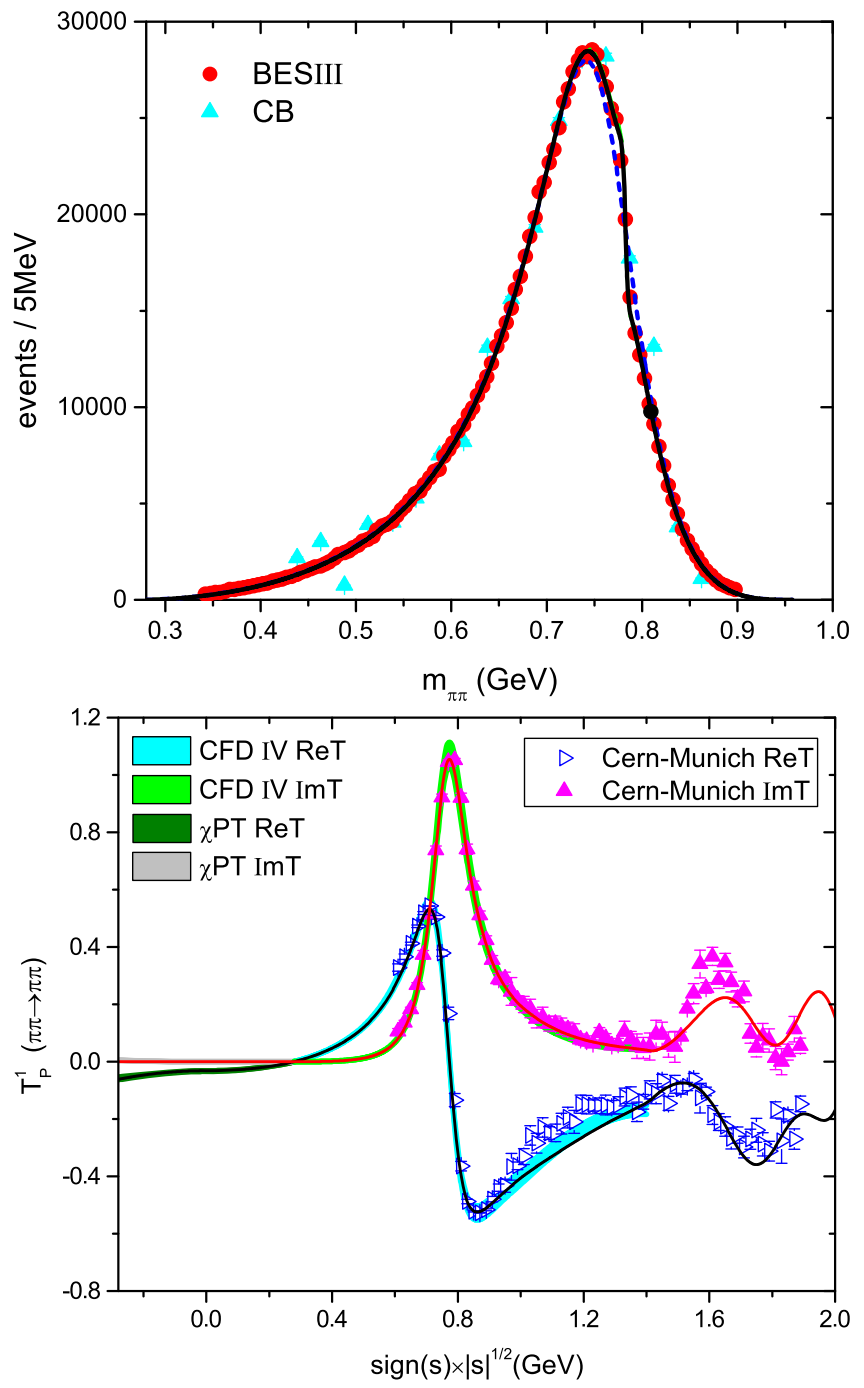

FIG. 3. The top figure corresponds to the fit to the invariant mass spectrum of $\eta^{\prime} \rightarrow \pi \pi \gamma$. The black solid line is the one of fit 3 , the blue dotted line is of fit 1 . As explained in the text, fit 2 is almost indistinguishable from fit 3 and thus not shown. The BESIII data is from [27] and Crystal Ball data from [24]. The bottom figure corresponds to the fit to the experiment data of the $\pi \pi \rightarrow \pi \pi P$ wave. The Cern-Munich data are from [58], and the olive and light grey bands in the low energy region are from Refs. [66-69]. 
TABLE II. The parameters of the $\pi \pi \rightarrow \pi \pi$ P-wave, given in Eq. (25). The uncertainty is from MINUIT. The unit of $c_{j}$ is $\mathrm{GeV}^{-2 j}$.

\begin{tabular}{llll}
\hline \hline$c_{0}=0.4283$ & $c_{1}=-0.2959$ & $c_{2}=0.6173(16)$ & $c_{3}=-0.7092(11)$ \\
$c_{4}=0.3774(4)$ & $c_{5}=-0.0909(1)$ & $c_{6}=0.0081(1)$ & \\
\hline \hline
\end{tabular}

to the BESIII data is much better. The invariant mass spectrum of fit 2 is almost indistinguishable from that of fit 3; thus we do not show it in Fig. 3. However, the decay width of $\eta^{\prime} \rightarrow \pi^{+} \pi^{-} \gamma$, which is $38.9 \pm 8.2 \mathrm{keV}$ now, deviates from the value of PDG [61] by $32 \%$. The anomaly is estimated to be $4.36 \mathrm{GeV}^{-3}$. For fit 3, the fit is rather good. The fit quality is improved both in the low energy region and the $\rho-\omega$ mixing region, compared to fit 2 . This is reasonable as, by tuning the free parameter $\alpha_{0}$, the amplitude at low energies can be adjusted, and the same holds for the isospin violation part in the high-energy region. We notice that $\alpha_{0}$ is shifted by $28 \%$ compared to that of fit 2, which also quantifies how much the anomaly can affect the fit. Consequently, the anomaly is $5.61 \pm 0.29 \mathrm{GeV}^{-3}$, shifted by $29 \%$ from the one given by matching to the tree-level amplitude of the WZW term. Since the tree-level amplitude is calculated in the large $N_{C}$ expansion, a typical $1 / N_{C}$ correction is reasonable. Considering also the improved fit quality of fit 3 with respect to fit 2, the correction of the order $1 / N_{C}$ to the treelevel amplitude of the WZW term in an anomalous decay process, is not only reasonable but also necessary. The normalization factor in fit 3 is similar to the one in fit 1 but decreases a lot compared to that of fit 2 . The reason is that, when $\alpha_{0}$ and $\alpha_{1}$ increase, the normalization factor has to decrease so as to compensate for the amplitude $F_{1+}^{1}(s)$. Comparing the quality of these fits, we consider fit 3 to be the reference result.

In the next section, in order to extract the couplings of the resonances, the $P$-wave $\pi \pi$ scattering amplitude is needed in addition to the decay amplitude of $\eta^{\prime} \rightarrow \pi^{+} \pi^{-} \gamma$ described above. To get the $P$-wave $\pi \pi$ scattering amplitude with isospin $I=1$, we adopt the following representation:

$$
T_{P}^{1}(s)=\left(s-4 M_{\pi}^{2}\right) \Omega_{1}^{1}(s) \sum_{i=0}^{6} c_{i}\left(s-4 M_{\pi}^{2}\right)^{i},
$$

with the $c_{i}$ unknown constants. The two constants, $c_{0}$ and $c_{1}$, are fixed by the relevant threshold parameters: scattering length $\sim 0.0387 \pm 0.0012 M_{\pi}^{-3}$ and slope parameter $\sim 0.0051 \pm 0.0026 M_{\pi}^{-5}$ [57]. The Cern-Munich data [58], CFDIV amplitude [57], and the amplitude from the Roy equation analysis $[56]^{4}$ on the complex $s$-plane are fitted to pin down the other constants. Finally we have all the values

\footnotetext{
${ }^{4}$ We replace the phase in the energy region of $0.8-1.4 \mathrm{GeV}$ by more recent analysis [42,57]. The results are in agreement with that of the original paper [56].
}

for the parameters, collected in Table II. The resulting $T_{P}^{1}(s)$ amplitude on the real axis is shown in the bottom panel of Fig. 3. Its analytic continuation to the complex $s$-plane, confronted with that of the Roy equation analysis [56], is shown in Fig. 4. We do not plot the amplitude on the upper half of $s$-plane, as it is readily obtainable from the one on the lower half of the $s$-plane according to the Schwarz reflection principle. The contribution of the 1.h.c to the shade region of the $s$-plane, as shown in Fig. 4, is properly implemented by fitting to the data as well as the amplitude of the Roy equation in the presence of the crossing symmetry. We see that our amplitudes are quite similar to the ones from the Roy equation analysis on the complex $s$-plane. The distribution of contours is in good agreement with each other. Moreover, their gradient variations, the shading of the color from blue to red, are compatible. Nevertheless, amplitudes on the edge of the domain, shown in Fig. 4, are less consistent with a difference $\leq 0.1$. This means our $T_{P}^{1}(s)$ amplitude is constrained rather well on the complex plane, allowing for a reliable extraction of the poles and residues.

\section{B. Couplings of $\boldsymbol{g}_{\eta^{\prime} V \gamma}$}

With a specific amplitude, the couplings of a resonance are defined by the residues of the pole on the complex $s$-plane. Based on the results of fit 3 , the absolute values of

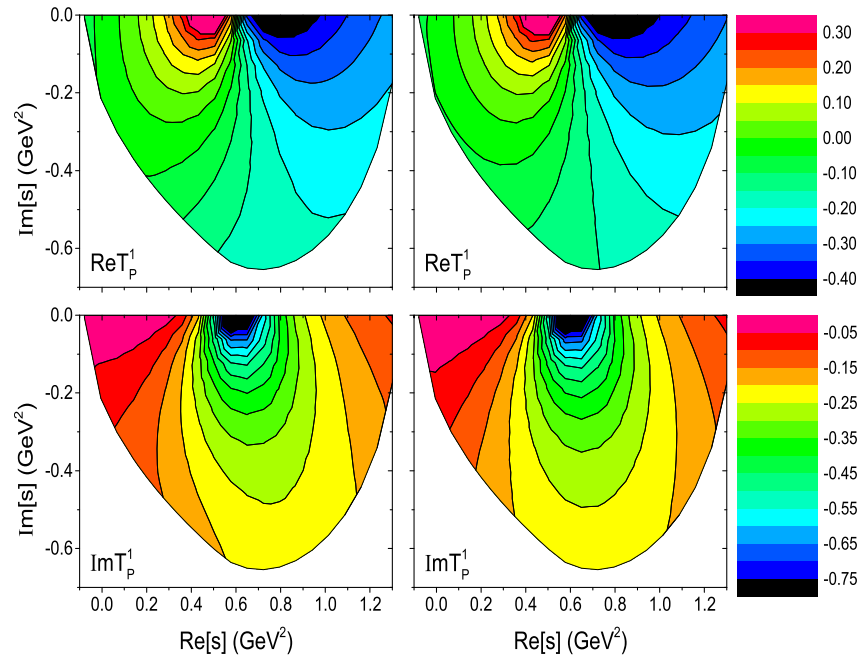

FIG. 4. Comparison of our amplitudes with the ones from the Roy equation analysis in the domain where the Roy equations work. On the left side are real and imaginary parts of our amplitudes, and on the right side are those from the Roy equation [56]. 
TABLE III. Predictions based on fit 3, as explained in the text. The following PDG [61] values are used: $\Gamma\left(\eta^{\prime} \rightarrow \gamma \pi^{+} \pi^{-}\right)=$ $57.3 \pm 1.0 \mathrm{keV}$ and $\Gamma\left(\eta^{\prime} \rightarrow \omega \gamma\right)=5.16 \pm 0.26 \mathrm{keV}$

\begin{tabular}{lcccccc}
\hline \hline State & Pole location $\mathrm{MeV}$ & $\left|g_{V \pi \pi}\right| \mathrm{MeV}$ & $\left|g_{\eta^{\prime} V}\right| \mathrm{MeV}$ & $\Gamma(V \rightarrow \pi \pi) \mathrm{MeV}$ & $\Gamma\left(\eta^{\prime} \rightarrow X \gamma\right) \mathrm{keV}$ & $\Gamma\left(\eta^{\prime} \rightarrow \pi^{+} \pi^{-} \gamma\right) \mathrm{keV}$ \\
\hline$\rho$ & $762.7(23)-\mathrm{i} 68.3(55)$ & $340.1(60)$ & $20.1(9)$ & $141.2(53)$ & $56.6(53)$ & $56.6(53)$ \\
$\omega$ & $782.56(12)-\mathrm{i} 4.24(4)$ & $10.4(4)$ & $5.68(74)$ & $0.130(1)$ & $4.10(97)$ & $0.0675(160)$ \\
A(anomaly) & $\cdots$ & $\cdots$ & $\cdots$ & $\cdots$ & $\cdots$ & $3.34(35)$ \\
Total & $\cdots$ & - & $\cdots$ & $\cdots$ & $\cdots$ & $62.0(59)$ \\
\hline \hline
\end{tabular}

the couplings $g_{\eta^{\prime} V \gamma}, V \in\{\rho, \omega\}$, extracted on the appropriate Riemann sheets are compiled in Table III.

The definition of the couplings on the appropriate Riemann sheet is given as

$$
M_{1+}^{1 \mathrm{II}}(s)=\frac{e g_{\eta^{\prime} V \gamma} g_{V \pi \pi}}{s_{V}-s}, \quad T_{P}^{1 \mathrm{II}}(s)=\frac{g_{V \pi \pi}^{2}}{s_{V}-s},
$$

where "II" denotes the second Riemann sheet. $M_{1+}^{1}$ is the $\eta^{\prime} \rightarrow \pi \pi \gamma$ decay amplitude given in Eq. (2), and $T_{P}^{1}(s)$ is the $P$-wave $\pi \pi \rightarrow \pi \pi$ scattering amplitude from Eq. (25). What those couplings mean in terms of decay width is provided as

$$
\begin{gathered}
\Gamma\left(\eta^{\prime} \rightarrow V \gamma\right)=\frac{16 \pi \alpha\left(M_{\eta^{\prime}}^{2}-M_{V}^{2}\right)\left|g_{\eta^{\prime} V \gamma}\right|^{2}}{M_{\eta^{\prime}}^{3}}, \\
\Gamma(V \rightarrow \pi \pi)=\frac{\rho\left(M_{V}^{2}\right)\left|g_{V \pi \pi}\right|^{2}}{M_{V}},
\end{gathered}
$$

with $\alpha$ the usual QED fine structure constant.

To obtain the couplings of $\omega$, we adopt the standard Blatt-Weisskopf barrier factor representation [42]

$$
g_{\omega \pi \pi}^{2}(s)=\frac{M_{\omega} \Gamma_{\omega} \mathrm{BR}_{\omega \rightarrow \pi \pi} \mathcal{Q}\left(M_{\omega}^{2}\right)}{\rho\left(M_{\omega}^{2}\right) \mathcal{Q}(s)},
$$

with $\mathcal{Q}(s)=1+q^{2} /\left(s-4 M_{\pi}^{2}\right)$, and $q$ is chosen to be $1 \mathrm{GeV}$. The mass, width and $\pi \pi$-mode branching ratio of the $\omega$ are taken from the PDG [61]: $M_{\omega}=782.65 \pm$ $0.12 \mathrm{MeV}, \Gamma_{\omega}=8.49 \pm 0.08 \mathrm{MeV}$ and $\mathrm{BR}_{\omega \rightarrow \pi \pi}=0.0153 \pm$ 0.0012 . Notice that the width is rather small, and thus we can ignore its energy dependence 5 To proceed, we define the coupling $g_{\eta^{\prime} \omega \gamma}$ through

\footnotetext{
${ }^{5}$ In a more dedicated way, one can use the standard BreitWigner formalism to represent the $\pi \pi \rightarrow \omega \rightarrow \pi \pi$ amplitude [42],

$$
T_{P \omega}^{1}=\frac{g_{2}(s)^{2}}{M^{2}-s-i \rho_{1}(s) g_{1}^{2}(s)-i \rho_{2}(s) g_{2}^{2}(s)-i \rho_{3}(s) g_{3}^{2}(s)} .
$$

Here 1,2, 3 represents the $\pi \gamma, \pi \pi$ and $\pi \pi \pi$ channels, respectively. Following it one can extract out the pole and residue on the $(-,-,-)$ plane. As we have checked, the results obtained in this way are quite the same as what we obtained in Table III.
}

$$
g_{\eta^{\prime} \omega \gamma}=-\frac{\beta_{0}\left(M_{\eta^{\prime}}^{2}-s_{\omega}\right) \sqrt{3 s_{\omega}} \rho\left(s_{\omega}\right)\left(F_{c}+F_{d}\right)_{s_{\omega}} \Omega_{1}^{1}\left(s_{\omega}\right)}{256 \pi \mathrm{BW}\left[\omega, s_{\omega}\right] g_{\omega \pi \pi}\left(s_{\omega}\right)},
$$

where $s_{\omega}=M_{\omega}^{2}-i M_{\omega} \Gamma_{\omega}$ and $\mathrm{BW}[\omega, s]$ is the BreitWigner representation given in Eq. (A7).

To get the anomaly contribution to the width of $\Gamma\left(\eta^{\prime} \rightarrow\right.$ $\pi^{+} \pi^{-} \gamma$ ), for simplicity we use $F_{1+}^{1}(0)$ instead of $F_{1+}^{1}(s)$, when integrating over $\sqrt{s}$ in Eq. (22). The differential decay width of the mode $\eta^{\prime} \rightarrow \omega \gamma \rightarrow \pi^{+} \pi^{-} \gamma$ is written as

$$
\frac{d \Gamma}{d \sqrt{s}}=\frac{3 \alpha s^{3 / 2} \rho(s)^{3}\left(M_{\eta^{\prime}}^{2}-s\right)^{3}\left|\beta_{0}\left(F_{c}+F_{d}\right) \Omega_{1}^{1}(s)\right|^{2}}{2048 \pi^{2} M_{\eta^{\prime}}^{3}} .
$$

The contribution through the intermediate $\rho$ meson is the same as $\Gamma\left(\eta^{\prime} \rightarrow \rho \gamma\right)$ since $\rho$ decays into $\pi \pi$ to $100 \%$, see also Eq. (32). Finally, we obtain the poles and couplings, based on fit 3 , and compute the decay widths with the help of Eqs. (27), (28) and (31). The results are shown in Table III. Our uncertainty is from the fit, combined the error from MINUIT and the systematic one: the correlation between the coefficients, see Eqs. (21), (25), and the uncertainty of the phase in the Omnès function. Our estimation shows that the systematic error dominates the uncertainty.

We notice that the pole position of $\rho$ is compatible with the one obtained by Padé approximants in [70]. The $\rho$ pole position is shifted a bit compared to the Breit-Wigner mass and width given by PDG [61], while this is not the case for $\omega$. The reason is that $\rho$ is much wider and the pole is farther away from the real axis, thus "narrow resonance approximation" is not good enough to describe the amplitude. With the pole locations one can extract out the residues and thus determine the contribution to $\Gamma\left(\eta^{\prime} \rightarrow \pi^{+} \pi^{-} \gamma\right)$ from each resonance, separately.

We obtain $\Gamma\left(\eta^{\prime} \rightarrow \omega \gamma \rightarrow \pi^{+} \pi^{-} \gamma\right)=67.5 \pm 16.0 \mathrm{eV}$. Since the $\omega$ is rather narrow, we can use the sequential decay formula $[71,72]$ to do a cross-check. The decay width in this way is given by

$$
\Gamma\left(\eta^{\prime} \rightarrow \omega \gamma \rightarrow \pi \pi \gamma\right)=\frac{\Gamma\left(\eta^{\prime} \rightarrow \omega \gamma\right) \Gamma(\omega \rightarrow \pi \pi)}{\Gamma_{\omega}} .
$$

In combination with Eqs. (28) and (29), we obtain $\Gamma\left(\eta^{\prime} \rightarrow \omega \gamma \rightarrow \pi^{+} \pi^{-} \gamma\right)=62.8 \pm 19.8 \mathrm{eV}$, which is in $\operatorname{good}$ agreement with the one calculated from Eq. (31). With $\Gamma\left(\eta^{\prime} \rightarrow \omega \gamma \rightarrow \pi^{+} \pi^{-} \gamma\right)=67.5 \pm 16.0 \mathrm{eV}$ and $\Gamma(\omega \rightarrow \pi \pi)=$ $0.130 \pm 0.010 \mathrm{MeV}$ taken from PDG, we obtain 
$\Gamma\left(\eta^{\prime} \rightarrow \omega \gamma\right)=4.41 \pm 1.04 \mathrm{keV}$. This is rather close to the value of $4.10 \pm 0.97 \mathrm{keV}$, obtained in the way described by Eqs. (27) and (30). Comparing these two decay widths, we notice that the one given by Eqs. (31), (32) contains the more dedicated energy dependence and is closer to that of PDG; thus we adopt $\Gamma\left(\eta^{\prime} \rightarrow \omega \gamma\right)=4.41 \pm 1.04 \mathrm{keV}$ as the optimal one.

It should be pointed out that $g_{\eta^{\prime} \omega \gamma}$ is correlated to $g_{\omega \pi \pi}\left(s_{\omega}\right)$, as can be seen from Eq. (30). Such a correlation is propagated to the decay widths, see Eq. (32). With $\Gamma\left(\eta^{\prime} \rightarrow \omega \gamma \rightarrow \pi^{+} \pi^{-} \gamma\right)=67.5 \pm 16.0 \mathrm{eV}$, if we fix $\Gamma\left(\eta^{\prime} \rightarrow \omega \gamma\right)=5.16 \pm 0.26 \mathrm{keV}$ instead of $\Gamma(\omega \rightarrow \pi \pi)$, we get $\Gamma(\omega \rightarrow \pi \pi)=0.111 \pm 0.026 \mathrm{MeV}$ or $\mathrm{BR}_{\omega \rightarrow \pi \pi}=$ $1.31 \pm 0.31 \%$ from Eq. (32). This is compatible with the previous analysis given by Ref. [5], shown in Table II therein.

The contributions to the decay width of $\Gamma\left(\eta^{\prime} \rightarrow \pi^{+} \pi^{-} \gamma\right)$, induced by an intermediate $\rho$ and $\omega$ as well as an anomaly "A", are given as follows: $56.6 \pm 5.3 \mathrm{keV}$ through $\rho$, $67.5 \pm 16.0 \mathrm{eV}$ through $\omega$, and $3.34 \pm 0.35 \mathrm{keV}$ through the anomaly. The contact isospin-violating term, shown in Fig. 2(b), contributes to the anomaly part too. However, its contribution is tiny and hence neglected. It is found that the $\rho$ dominates the contribution, and the anomaly contributes more than the $\omega$. This is not surprising as the $\rho$ dominates the $P$-wave and the anomaly contributes as a background in the whole energy region, while the $\omega$ only acts in a small region and rarely decays into $\pi \pi$.

For the decay widths of $\eta^{\prime} \rightarrow V \gamma$, we obtain $\Gamma\left(\eta^{\prime} \rightarrow \rho \gamma\right)=$ $56.6 \pm 5.3 \mathrm{keV}$, while in LO R $\chi \mathrm{T}$ [38] it is $53.7 \mathrm{keV}$. Likewise, $\Gamma\left(\eta^{\prime} \rightarrow \omega \gamma\right)=4.41 \pm 1.04 \mathrm{keV}$ [or $4.10 \pm 0.97$ through residue in Eqs. (27), (30)], while in LO $\mathrm{R} \chi \mathrm{T}$ it is $5.12 \mathrm{keV}$. Since in LO R $\chi \mathrm{T}$ the uncertainty is roughly $1 / 3$ when truncating the large $N_{C}$ expansion, the widths obtained here and in $\mathrm{LO} \mathrm{R} \chi \mathrm{T}$ are compatible if the errors are taken into

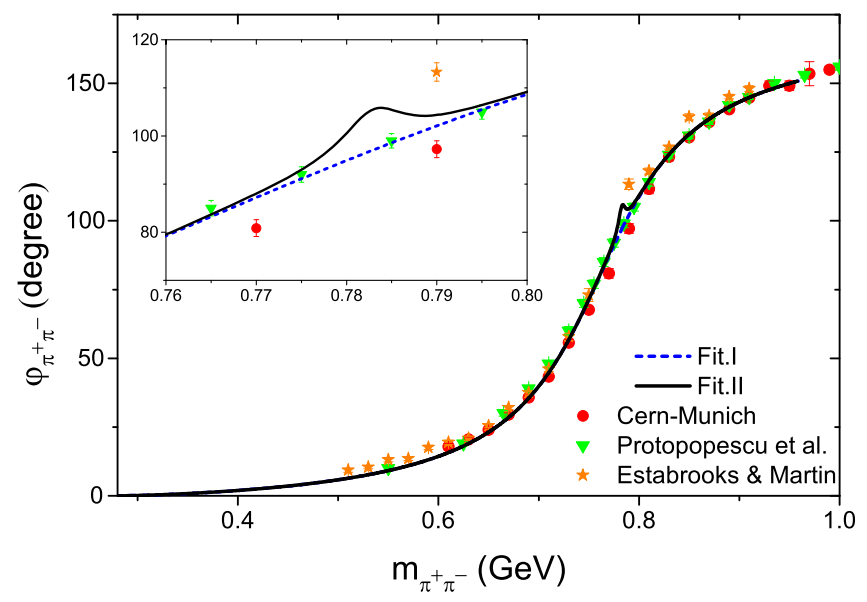

FIG. 5. Phase of $\pi^{+} \pi^{-} P$-wave from our amplitude analysis. The blue dashed line is from fit 1 and the black solid line from fit 3. The data sets of are from Ref. [58] (red circles), from Ref. [59] (green triangles) and from Ref. [60] (orange stars), respectively. account. This shows us how much the $\pi \pi$ FSI affects the strong interaction between the lightest vector and pseudoscalar mesons. Compared to the predictions given by $\mathrm{R} \chi \mathrm{T}$, the FSI effect is sizable but still within the uncertainty.

In addition, we find that in the whole kinematical region the phase of $\pi^{+} \pi^{-}$based on fit 3 is in good agreement with the one based on fit 1, i.e., the phase of $T_{P}^{1}(s)$, except for the energy region around $\sqrt{s}=M_{\omega}$, see Fig. 5. Obviously, there is a bump around the $\omega$ mass when taking into account the $\rho-\omega$ mixing. Note that there is no such bump for $\pi^{ \pm} \pi^{0}$, as the $\omega$ is an isospin singlet. It should be noted that for $\eta^{\prime} \rightarrow \pi^{+} \pi^{-} \gamma$ the 1.h.c (mainly from the $a_{2}$ ) and inelastic r.h.c (above $\bar{K} K$ threshold) are too far away from the energy region we are working. Thus the polynomial $P(s)$, in which the 1.h.c and r.h.c are absorbed, should be smooth in the physical energy region. This suggests that, to include isospin violation from the $\rho-\omega$ mixing in the line shape of $\eta^{\prime} \rightarrow \pi^{+} \pi^{-} \gamma$, it is more convenient to change the phase of $\pi^{+} \pi^{-}$in the Omnés function than to include a complicated complex form factor in $P(s)$ function. We notice that there is only one data point from [59] located at around $\sqrt{s}=M_{\omega}$, but it was obtained with a low resolution. Most experiments only take into account the isospin-conserved part (the contribution from the $\rho$ ) [58-60]. To reach a more definite conclusion on the phase caused by the isospin violation, a more accurate experimental measurement in the $\sqrt{s}=M_{\omega}$ region and a more careful theory analysis are required. Here we just point out that, through our amplitude analysis of the $\eta^{\prime} \rightarrow \pi^{+} \pi^{-} \gamma$ data provided by Ref. [27], there should be a bump of the $\pi^{+} \pi^{-} P$-wave phase around the energy point $\sqrt{s}=M_{\omega}$.

\section{CONCLUSION}

In this paper we have studied the process of $\eta^{\prime} \rightarrow \pi^{+} \pi^{-} \gamma$ by fitting to the latest invariant mass spectrum from BESIII [27]. The amplitude is constructed according to Watson's theorem, and the isospin-violating form factor is calculated within the framework of $\mathrm{R} \chi \mathrm{T}$ at $\mathrm{LO}$ in the $1 / N_{C}$ expansion. We find that the anomaly, defined in Eq. (23), is around $5.61 \pm 0.29 \mathrm{GeV}^{-3}$. It is shifted by an amount of $\mathcal{O}\left(1 / N_{C}\right)$ compared to the value calculated using the tree-level amplitude of the WZW term. The couplings and decay widths of the $\rho, \omega$ resonances are extracted properly, as shown in Table III. The contributions to $\Gamma\left(\eta^{\prime} \rightarrow \pi^{+} \pi^{-} \gamma\right)$ are quantified as follows: $\Gamma\left(\eta^{\prime} \rightarrow \rho \gamma \rightarrow \pi^{+} \pi^{-} \gamma\right)=$ $56.6 \pm 5.3 \mathrm{keV}, \Gamma\left(\eta^{\prime} \rightarrow \omega \gamma \rightarrow \pi^{+} \pi^{-} \gamma\right)=67.5 \pm 16.0 \mathrm{eV}$, and $\Gamma_{A}\left(\eta^{\prime} \rightarrow \pi^{+} \pi^{-} \gamma\right)=3.34 \pm 0.35 \mathrm{keV}$. The $\rho$ resonance dominates the intermediate process, while the anomaly contributes more than the $\omega$. We obtain $\Gamma\left(\eta^{\prime} \rightarrow \rho \gamma\right)=$ $56.6 \pm 5.3 \mathrm{keV}, \quad \Gamma\left(\eta^{\prime} \rightarrow \omega \gamma\right)=4.41 \pm 1.04 \mathrm{keV}$, which are consistent with the determinations from $\mathrm{LO} \mathrm{R} \chi \mathrm{T}$ within the uncertainty of around $1 / 3$. Finally we find the phase of the $P$-wave $\pi^{+} \pi^{-}$scattering amplitude should have a bump around $\sqrt{s}=M_{\omega}$. This work could be useful for the studies of strong interaction referring to the $\pi \pi$ final states, such as 
$J / \psi \rightarrow \gamma \pi \pi$ [73], $\bar{p} p \rightarrow X(3872) \rightarrow J / \psi \pi \pi$ in PANDA [74], and $B_{c}^{+} \rightarrow B_{s}^{0} \pi^{+} \pi^{0}[75]$.

\section{ACKNOWLEDGMENTS}

We are grateful to Zhi-Hui Guo for helpful discussions. We also wish to thank Chu-Wen Xiao for a careful reading of our manuscript and for his suggestions. This work is supported in part by the Deutsche Forschungsgemeinschaft (DFG) and the National Natural Science Foundation of China (NSFC) through funds provided to the Sino-German CRC 110 "Symmetries and the Emergence of Structure in QCD" (Grant No. TRR 110). The work of UGM was supported in part by The Chinese Academy of Sciences (CAS) President's International Fellowship Initiative (PIFI) with Grant No. 2017VMA0025. DLY thanks support from the Spanish Ministerio de Economía y Competitividad and the European Regional Development Fund, under Contracts No. FIS2014-51948-C2-1-P, No. FIS2014-51948-C2-2-P,
No. SEV-2014-0398 and by Generalitat Valenciana under Contract No. PROMETEOII/2014/0068.

\section{APPENDIX: ISOPIN-VIOLATING FORM FACTORS}

The amplitude of $\eta^{\prime} \rightarrow \pi^{+} \pi^{-} \gamma$ is given by

$$
\mathcal{M}_{\lambda}=e \epsilon^{\mu \nu \alpha \beta} \epsilon_{\mu}(k, \lambda) q_{\nu} p_{\alpha}^{+} p_{\beta}^{-} F(s) .
$$

For the form factor $F(s)$, the tree-level WZW anomaly term gives

$$
F_{a}=-\frac{N_{C}}{12 \sqrt{3} \pi^{2} F^{3}}\left(\sin \theta_{P}+\sqrt{2} \cos \theta_{P}\right) .
$$

The isospin-violating form factor is

$$
F_{\text {tree }}^{i . v}=F_{b}+F_{c}+F_{d},
$$

and

$$
\begin{aligned}
& F_{b}=-\frac{8 \sqrt{6} F_{V}\left(1+8 \sqrt{2} \alpha_{V} \frac{m_{\pi}^{2}}{M_{V}^{2}}\right)}{3 M_{V} F^{3}}\left(-\sin \delta+\frac{1}{\sqrt{3}} \cos \delta \sin \theta_{V}\right) \sin \delta\left(\sin \theta_{P}+\sqrt{2} \cos \theta_{P}\right) G_{R \eta^{\prime}}(0, s) B W_{R}[\omega, 0], \\
F_{c}= & \frac{4 \sqrt{2} G_{V}}{3 M_{V} F^{3}} \sin \delta\left\{\cos \delta \sin \theta_{V}\left[\sqrt{2} \cos \theta_{P}-\sin \theta_{P}\right]+\sqrt{2} \cos \delta \cos \theta_{V} \sin \theta_{P}-\sqrt{3} \sin \delta\left[\sqrt{2} \cos \theta_{P}+\sin \theta_{P}\right]\right\} \\
& \times C_{R \eta^{\prime} 1}\left(0, s, M_{\eta^{\prime}}^{2}\right) B W_{R}[\omega, s]+\frac{2 \sqrt{2} G_{V}}{18 M_{V} F^{3}} \sin \delta\left\{4 \cos \delta\left[-3 \cos \left(\theta_{V}-\theta_{P}\right)+\cos \left(\theta_{V}+\theta_{P}\right)+2 \sqrt{2} \sin \left(\theta_{V}+\theta_{P}\right)\right] m_{K}^{2}\right. \\
& \left.+\left[-6 \sqrt{3} \sin \delta\left[\sqrt{2} \cos \theta_{P}+\sin \theta_{P}\right]+\cos \delta\left[9 \cos \left(\theta_{V}-\theta_{P}\right)-\cos \left(\theta_{V}+\theta_{P}\right)-2 \sqrt{2} \sin \left(\theta_{V}+\theta_{P}\right)\right]\right] M_{\pi}^{2}\right\} C_{R \eta^{\prime} 2} B W_{R}[\omega, s],
\end{aligned}
$$

$$
\begin{aligned}
F_{d}= & -\frac{8 F_{V}\left(1+8 \sqrt{2} \alpha_{V} \frac{M_{\pi}^{2}}{M_{V}^{2}}\right) G_{V}}{\sqrt{6} F^{3}} \sin \delta\left(-\sin \delta+\frac{1}{\sqrt{3}} \cos \delta \sin \theta_{V}\right)\left(\sin ^{2} \delta\left(2 \cos \theta_{P}+\sqrt{2} \sin \theta_{P}\right)+\cos ^{2} \delta\left[2 \cos \theta_{P}+\sin \theta_{V}\left(4 \cos \theta_{V}\right.\right.\right. \\
& \left.\left.\left.-\sqrt{2} \sin \theta_{V}\right) \sin \theta_{P}\right]\right) D_{R \eta^{\prime} 1}\left(0, s, M_{\eta^{\prime}}^{2}\right) B W_{R R}[\omega, \omega, 0, s]-\frac{4 F_{V}\left(1+8 \sqrt{2} \alpha_{V} \frac{M_{\pi}^{2}}{M_{V}^{2}}\right) G_{V}}{3 \sqrt{6} F^{3}} \sin \delta\left(-\sin \delta+\frac{1}{\sqrt{3}} \cos \delta \sin \theta_{V}\right) \\
& \times\left\{4 \cos ^{2} \delta\left(\cos \theta_{P}\left[3-\cos 2 \theta_{V}-2 \sqrt{2} \sin 2 \theta_{V}\right]+\left[3 \sqrt{2}-\sqrt{2} \cos 2 \theta_{V}-4 \sin 2 \theta_{V}\right] \sin \theta_{P}\right) m_{K}^{2}+\left(6 \sin ^{2} \delta\left(2 \cos \theta_{P}+\sqrt{2} \sin \theta_{P}\right)\right.\right. \\
& \left.+\cos ^{2} \delta\left(4 \cos \left(2 \theta_{V}+\theta_{P}\right)+\sqrt{2}\left[8 \cos \theta_{P} \sin 2 \theta_{V}+\left(9-\cos 2 \theta_{V}\right) \sin \theta_{P}\right]\right) m_{\pi}^{2}\right\} D_{R \eta^{\prime} 2} B W_{R R}[\omega, \omega, 0, s] \\
& -\frac{2 F_{V}\left(1+8 \sqrt{2} \alpha_{V} M_{\pi}^{2}\right) G_{V}}{\sqrt{6} F^{3}} \sin \delta\left(\cos \delta+\frac{1}{\sqrt{3}} \sin \delta \sin \theta_{V}\right)\left\{\sin 2 \delta\left[-3 \sqrt{2}+\sqrt{2} \cos 2 \theta_{V}+4 \sin 2 \theta_{V}\right] \sin \theta_{P}\right\} \\
& \times D_{R \eta^{\prime} 1}\left(0, s, M_{\eta^{\prime}}^{2}\right) B W_{R R}[\rho, \omega, 0, s]-\frac{2 F_{V}\left(1+8 \sqrt{2} \alpha_{V} \frac{M_{\pi}^{2}}{M_{V}^{2}}\right) G_{V}}{3 \sqrt{6} F^{3}} \sin \delta\left(\cos \delta+\frac{1}{\sqrt{3}} \sin \delta \sin \theta_{V}\right) \\
& \times \sin 2 \delta\left\{-4 \cos \theta_{P}\left(-3+\cos 2 \theta_{V}+2 \sqrt{2} \sin 2 \theta_{V}\right)\left(m_{K}^{2}-m_{\pi}^{2}\right)+\left(-3 \sqrt{2}+\sqrt{2} \cos 2 \theta_{V}+4 \sin \left[2 \theta_{V}\right]\right) \sin \theta_{P}\left(4 m_{K}^{2}-m_{\pi}^{2}\right)\right\} \\
& \times D_{R \eta^{\prime} 2} B W_{R R}[\rho, \omega, 0, s]+\frac{2 F_{V}\left(1+8 \sqrt{2} \alpha_{V} \frac{2 m_{K}^{2}-M_{\pi}^{2}}{M_{V}^{2}}\right) G_{V} \sin 2 \delta\left\{\cos \theta_{V}\left(-4 \cos 2 \theta_{V}+\sqrt{2} \sin 2 \theta_{V}\right) \sin \theta_{P}\right\}}{3 \sqrt{2} F^{3}} \\
& \times D_{R \eta^{\prime} 1}\left(0, s, M_{\eta^{\prime}}^{2}\right) B W_{R R}[\phi, \omega, 0, s]-\frac{2 F_{V}\left(1+8 \sqrt{2} \alpha_{V} \frac{2 m_{K}^{2}-M_{\pi}^{2}}{M_{V}^{2}}\right) G_{V}}{9 \sqrt{2} F^{3}} \sin 2 \delta \cos \theta_{V}\left\{4 \cos \theta_{P}\left[2 \sqrt{2} \cos 2 \theta_{V}-\sin 2 \theta_{V}\right]\left(m_{K}^{2}-m_{\pi}^{2}\right)\right. \\
& \left.-\left(4 \cos 2 \theta_{V}-\sqrt{2} \sin 2 \theta_{V}\right) \sin \theta_{P}\left(4 m_{K}^{2}-m_{\pi}^{2}\right)\right\} D_{R \eta^{\prime} 2} B W_{R R}[\phi, \omega, 0, s],
\end{aligned}
$$


where

$$
\begin{aligned}
C_{R \eta^{\prime} 1}\left(Q^{2}, x, m^{2}\right)= & \left(c_{1}-c_{2}+c_{5}\right) Q^{2}-\left(c_{1}-c_{2}\right. \\
& \left.-c_{5}+2 c_{6}\right) x+\left(c_{1}+c_{2}-c_{5}\right) m^{2}, \\
C_{R \eta^{\prime} 2}= & 8 c_{3}, \\
D_{R \eta^{\prime} 1}\left(Q^{2}, x, m^{2}\right)= & d_{3}\left(Q^{2}+x\right)+\left(d_{1}-d_{3}\right) m^{2}, \\
D_{R \eta^{\prime} 2}= & 8 d_{2}, \\
G_{R \eta^{\prime}}\left(Q^{2}, s\right)= & \left(g_{1}+2 g_{2}-g_{3}\right)\left(s-2 m_{\pi}^{2}\right) \\
& +g_{2}\left(-Q^{2}+2 m_{\pi}^{2}+m_{\eta}^{2}\right)+\left(2 g_{4}+g_{5}\right) m_{\pi}^{2}, \\
B W_{R}[V, x] & =\frac{1}{M_{V}^{2}-i \Gamma_{V}(x) M_{V}-x}, \\
B W_{R R}\left[V_{1}, V_{2}, x, y\right] & =B W_{R}\left[V_{1}, x\right] B W_{R}\left[V_{2}, y\right] .
\end{aligned}
$$

Notice that $\Gamma_{V}(0)=0$. Here $\theta_{V}$ is the $\omega-\phi$ mixing angle, $\delta$ the $\rho-\omega$ mixing angle and $\theta_{P}$ the $\eta-\eta^{\prime}$ mixing angle. We follow the construction of vector resonance off shell widths in Ref. [76], where the parametrization of $\Gamma_{\rho}\left(q^{2}\right)$ is employed as

$$
\begin{aligned}
\Gamma_{\rho}\left(q^{2}\right)= & \frac{M_{\rho} q^{2}}{96 \pi F^{2}}\left[\left(1-\frac{4 M_{\pi}^{2}}{q^{2}}\right)^{\frac{3}{2}} \theta\left(q^{2}-4 M_{\pi}^{2}\right)\right. \\
& \left.+\frac{1}{2}\left(1-\frac{4 m_{K}^{2}}{q^{2}}\right)^{\frac{3}{2}} \theta\left(q^{2}-4 m_{K}^{2}\right)\right],
\end{aligned}
$$

with $F$ the pion decay constant. For $\omega, \phi$ width we use constant decay widths. The resonance parameters are given by PDG [61], and all other parameters can be found in fit 4 of Ref. [38]. For convenience we compile them in Table IV. The form factors of $F_{\eta^{\prime} \rightarrow \rho \gamma}$ and $F_{\eta^{\prime} \rightarrow \omega \gamma}$ can be found in [38], too. From the matching between $\mathrm{R} \chi \mathrm{T}$ and $\mathrm{QCD}$ one can find the constraints on the unknown couplings [34,37]:
TABLE IV. Parameters for $\rho-\omega$ mixing amplitudes.

\begin{tabular}{lcrr}
\hline \hline$F_{V}(\mathrm{GeV})$ & $0.148 \pm 0.001$ & \multicolumn{1}{c}{$\alpha_{V}$} & $0.0126 \pm 0.0007$ \\
\hline $2 g_{4}+g_{5}$ & $-0.493 \pm 0.003$ & $\theta_{V}\left({ }^{\circ}\right)$ & $38.94 \pm 0.02$ \\
$d_{2}$ & $0.0359 \pm 0.0007$ & $\theta_{P}\left({ }^{\circ}\right)$ & $-21.37 \pm 0.26$ \\
$c_{3}$ & $0.00689 \pm 0.00017$ & $\delta\left(^{\circ}\right)$ & $2.12 \pm 0.06$ \\
\hline \hline
\end{tabular}

$$
\begin{aligned}
F_{V} G_{V} & =F^{2}, \\
d_{1}+8 d_{2}-d_{3} & =\frac{F^{2}}{8 F_{V}^{2}}, \\
4 c_{3}+c_{1} & =0, \\
d_{3} & =-\frac{N_{C}}{192 \pi^{2}} \frac{M_{V}^{2}}{F_{V} G_{V}}, \\
c_{1}-c_{2}+c_{5} & =0, \\
g_{2} & =\frac{N_{C}}{192 \sqrt{2} \pi^{2}} \frac{M_{V}}{F_{V}}, \\
c_{1}+c_{2}+8 c_{3}-c_{5} & =0, \\
g_{1}-g_{3} & =-\frac{N_{C}}{96 \sqrt{2} \pi^{2}} \frac{M_{V}}{F_{V}}, \\
c_{1}-c_{2}-c_{5}+2 c_{6} & =-\frac{N_{C}}{96 \sqrt{2} \pi^{2}} \frac{M_{V}}{G_{V}} .
\end{aligned}
$$

We notice that these constraints are not the same as a more complicated one [77], where heavier pseudoscalar resonances are included. Since we only focus on the physics below $\sqrt{s}=M_{\eta^{\prime}}$, we do not take the heavier resonances into consideration. It would be interesting to note that our $F_{V}$ is closer to $F_{V}=\sqrt{3} F[78,79]$ rather than $F_{V}=\sqrt{2} F$ [33]. The former constraint is from the combined analysis of an axial-vector current with the contribution from three pseudoscalars $\left(\tau \rightarrow K K \pi \nu_{\tau}\right)$ and $\tau \rightarrow \gamma P \nu_{\tau}$ (or $\left.\langle V P P\rangle\right)$, while our constraints are based on the analysis of a vector current with the contribution from three pseudoscalars $\left(e^{+} e^{-} \rightarrow \pi^{+} \pi^{-} \pi^{0} / \eta\right)$. A more careful study is needed for a good understanding of the short-distance QCD constraint.
[1] R. A. Bertlmann, Anomalies in Quantum Field Theory, International Series of Monographs on Physics Vol. 19 (Clarendon, Oxford, UK, 1996), p. 566.

[2] F. Stollenwerk, C. Hanhart, A. Kupść, U.-G. Meißner, and A. Wirzba, Phys. Lett. B 707, 184 (2012).

[3] B. Kubis and J. Plenter, Eur. Phys. J. C 75, 283 (2015).

[4] C. W. Xiao, T. Dato, C. Hanhart, B. Kubis, U.-G. Meißner, and A. Wirzba, arXiv:1509.02194.
[5] C. Hanhart, S. Holz, B. Kubis, A. Kupść, A. Wirzba, and C. W. Xiao, Eur. Phys. J. C 77, 98 (2017).

[6] V. Pascalutsa and M. Vanderhaeghen, Phys. Rev. Lett. 105, 201603 (2010).

[7] I. Danilkin and M. Vanderhaeghen, Phys. Rev. D 95, 014019 (2017).

[8] L. Y. Dai and M. R. Pennington, Phys. Rev. D 95, 056007 (2017). 
[9] T. Blum, P. A. Boyle, T. Izubuchi, L. Jin, A. Jüttner, C. Lehner, K. Maltman, M. Marinkovic, A. Portelli, and M. Spraggs, Phys. Rev. Lett. 116, 232002 (2016).

[10] F. Jegerlehner, Springer Tracts Mod. Phys. 274, 1 (2017).

[11] M. Della Morte, A. Francis, V. Gülpers, G. Herdoíza, G. von Hippel, H. Horch, B. Jäger, H. B. Meyer, A. Nyffeler, and H. Wittig, J. High Energy Phys. 10 (2017) 020.

[12] R. M. Carey et al., Report No. FERMILAB-PROPOSAL0989, 2009; B. C. K. Casey, AIP Conf. Proc. 1182, 726 (2009).

[13] N. Saito (J-PARC g-2/EDM Collaboration), AIP Conf. Proc. 1467, 45 (2012).

[14] P. Adlarson et al. (WASA-at-COSY Collaboration), Phys. Lett. B 707, 243 (2012).

[15] D. Babusci et al. (KLOE Collaboration), Phys. Lett. B 718, 910 (2013).

[16] L. W. Bartel et al. (JADE Collaboration), Phys. Lett. 113B, 190 (1982).

[17] H. Behrends et al. (CELLO Collaboration), Phys. Lett. 114B, 378 (1982); 125B, 518(E) (1983).

[18] C. Berger et al. (LUTO Collaboration), Phys. Lett. 142B, 125 (1984).

[19] M. Althoff et al. (TASSO Collaboration), Phys. Lett. 147B, 487 (1984).

[20] H. Aihara et al. (TPC Collaboration), Phys. Rev. D 35, 2650 (1987).

[21] H. Albrecht et al. (ARGUS Collaboration), Phys. Lett. B 199, 457 (1987).

[22] X. W. Kang, B. Kubis, C. Hanhart, and U. G. Meißner, Phys. Rev. D 89, 053015 (2014).

[23] S. I. Bityukov et al., Z. Phys. C 50, 451 (1991).

[24] A. Abele et al. (Crystal Barrel Collaboration), Phys. Lett. B 402, 195 (1997).

[25] M. Acciarri et al. (L3 Collaboration), Phys. Lett. B 418, 399 (1998).

[26] M. Benayoun, P. David, L. DelBuono, and O. Leitner, Eur. Phys. J. C 65, 211 (2010).

[27] M. Ablikim et al. (BESIII Collaboration), arXiv:1712 .01525 .

[28] S. S. Fang, A. Kupsc, and D. H. Wei, arXiv:1710.05173.

[29] J. Wess and B. Zumino, Phys. Lett. 37B, 95 (1971).

[30] E. Witten, Nucl. Phys. B223, 422 (1983).

[31] B. Borasoy and R. Nissler, Nucl. Phys. A740, 362 (2004).

[32] G. Ecker, J. Gasser, A. Pich, and E. de Rafael, Nucl. Phys. B321, 311 (1989).

[33] G. Ecker, J. Gasser, H. Leutwyler, A. Pich, and E. de Rafael, Phys. Lett. B 223, 425 (1989).

[34] P. Ruiz Femenía, A. Pich, and J. Portolés, J. High Energy Phys. 07 (2003) 003.

[35] V. Cirigliano, G. Ecker, M. Eidemuller, R. Kaiser, A. Pich, and J. Portoles, Nucl. Phys. B753, 139 (2006).

[36] Z. H. Guo, J. J. Sanz-Cillero, and H. Q. Zheng, J. High Energy Phys. 06 (2007) 030.

[37] D. Gómez Dumm, P. Roig, A. Pich, and J. Portolés, Phys. Rev. D 81, 034031 (2010).

[38] L. Y. Dai, J. Portolés, and O. Shekhovtsova, Phys. Rev. D 88, 056001 (2013).

[39] R. Urech, Phys. Lett. B 355, 308 (1995).
[40] A. Kucukarslan and U.-G. Meißner, Mod. Phys. Lett. A 21, 1423 (2006).

[41] R. Garcia-Martin and B. Moussallam, Eur. Phys. J. C 70, 155 (2010).

[42] L. Y. Dai and M. R. Pennington, Phys. Lett. B 736, 11 (2014); Phys. Rev. D 90, 036004 (2014).

[43] P. Guo, I. V. Danilkin, D. Schott, C. Fernndez-Ramrez, V. Mathieu, and A. P. Szczepaniak, Phys. Rev. D 92, 054016 (2015).

[44] S. P. Schneider, B. Kubis, and F. Niecknig, Phys. Rev. D 86, 054013 (2012).

[45] D. L. Yao, M. Albaladejo, P. Fernndez-Soler, F. K. Guo, and J. Nieves, arXiv:1711.11515.

[46] M. Bando, T. Kugo, and K. Yamawaki, Phys. Rep. 164, 217 (1988).

[47] U.-G. Meißner, Phys. Rep. 161, 213 (1988).

[48] L. Y. Dai, V. Mathieu, E. Passemar, M. R. Pennington, and A. Szczepaniak (to be published).

[49] L. Y. Dai and U.-G. Meißner, arXiv:1706.10123.

[50] H. Leutwyler, Nucl. Phys. B, Proc. Suppl. 64, 223 (1998).

[51] T. Feldmann, P. Kroll, and B. Stech, Phys. Rev. D 58, 114006 (1998).

[52] R. Kaiser and H. Leutwyler, in Nonperturbative methods in quantum field theory, Adelaide, 1998, p. 15, [arXiv: hep-ph/ 9806336].

[53] X. K. Guo, Z. H. Guo, J. A. Oller, and J. J. Sanz-Cillero, J. High Energy Phys. 06 (2015) 175.

[54] Y. H. Chen, Z. H. Guo, and H. Q. Zheng, Phys. Rev. D 85, 054018 (2012).

[55] Y. H. Chen, D. L. Yao, and H. Q. Zheng, Commun. Theor. Phys. 69, 50 (2018).

[56] G. Colangelo, J. Gasser, and H. Leutwyler, Nucl. Phys. B603, 195 (2002).

[57] R. García- Martín, R. Kamiński, J. R. Peláez, J. Ruiz de Elvira, and F. J. Ynduráin, Phys. Rev. D 83, 074004 (2011).

[58] B. Hyams et al., Nucl. Phys. B64, 134 (1973); G. Grayer et al., Nucl. Phys. B75, 189 (1974); B. Hyams et al., Nucl. Phys. B100, 205 (1975).

[59] S. D. Protopopescu, M. Alston-Garnjost, A. Barbaro-Galtieri, S. M. Flatté, J. H. Friedman, T. A. Lasinski, G. R. Lynch, M. S. Rabin, and F. T. Solmitz, Phys. Rev. D 7, 1279 (1973).

[60] P. Estabrooks and A. D. Martin, Nucl. Phys. B79, 301 (1974).

[61] C. Patrignani et al. (PDG Collaboration), Chin. Phys. C 40, 100001 (2016).

[62] L. Y. Dai, M. Shi, G. Y. Tang, and H. Q. Zheng, Phys. Rev. D 92, 014020 (2015).

[63] H. Y. Cheng and X. W. Kang, Eur. Phys. J. C 77, 587 (2017).

[64] F. Ambrosino et al. (KLOE Collaboration), Phys. Lett. B648, 267 (2007).

[65] J. Hietala, D. Cronin-Hennessy, T. Pedlar, and I. Shipsey, Phys. Rev. D 92, 012009 (2015).

[66] J. Gasser and H. Leutwyler, Nucl. Phys. B250, 465 (1985).

[67] J. Bijnens, G. Colangelo, and J. Gasser, Nucl. Phys. B427, 427 (1994).

[68] A. G. Nicola and J. R. Pelaez, Phys. Rev. D 65, 054009 (2002). 
[69] L. Y. Dai, X. G. Wang, and H. Q. Zheng, Commun. Theor. Phys. 57, 841 (2012); 58, 410 (2012).

[70] P. Masjuan, J. Ruiz de Elvira, and J. J. Sanz-Cillero, Phys. Rev. D 90, 097901 (2014).

[71] X. W. Kang and J. A. Oller, Eur. Phys. J. C 77, 399 (2017).

[72] H. Y. Cheng and C. W. Chiang, Phys. Rev. D 81, 074031 (2010).

[73] M. Ablikim et al. (BES Collaboration), Phys. Lett. B 642, 441 (2006).
[74] E. Prencipe et al. (PANDA Collaboration), Nucl. Part. Phys. Proc. 273-275, 231 (2016).

[75] X.-H. Liu and U.-G. Meißner, Eur. Phys. J. C 77, 816 (2017).

[76] D. Gómez Dumm, A. Pich, and J. Portolés, Phys. Rev. D 62 , 054014 (2000).

[77] K. Kampf and J. Novotny, Phys. Rev. D 84, 014036 (2011).

[78] Z.H. Guo and P. Roig, Phys. Rev. D 82, 113016 (2010).

[79] P. Roig and J. J. Sanz Cillero, Phys. Lett. B 733, 158 (2014). 